\title{
Human Face Detection by using Skin Color Segmentation, Face Features and Regions Properties
}

\author{
Devendra Singh Raghuvanshi \\ M.Tech \\ MANIT Bhopal
}

\author{
Dheeraj Agrawal \\ Professor \\ MANIT Bhopal
}

\begin{abstract}
The goal of face detection is to locate all regions that contain a face. This paper has a simple face detection procedure which has two major steps, first to segment skin region from an image, and second, to decide these regions contain human face or not. Our procedure is based on skin color segmentation and human face features (knowledgebased approach). In this paper, we used $\mathrm{RGB}, \mathrm{YC}_{\mathrm{b}} \mathrm{C}_{\mathrm{r}}$, CEILAB $(L * a * b)$ and HSV color models for skin color segmentation. These color models with thresholds, help to remove non skin like pixels from an image. We tested each skin region, that skin region is actually represents a human face or not, by using human face features based on knowledge of geometrical properties of human face. The experiment result shows that, the algorithm gives hopeful results. At last, we concluded this paper and proposed future work.
\end{abstract}

\section{INTRODUCTION}

Face detection is the problem of determining whether a sub-window of an image contains a face. Several applications, such as face processing (i.e. face, expression, and gesture recognition), computer human interaction, human crowd surveillance, biometric, video surveillance, artificial intelligence and content-based image retrieval [1]. All of these applications, stated above, require face

\begin{abstract}
detection, which can be simply viewed as a preprocessing step, for obtaining the 'object'. In other words, many of the techniques are proposed for these applications assume that, the location of the face is pre-identified and available for the next step. First problem is come in the way of face detection, is chosen proper color model for skin color segmentation. There are several color models and each has specific work field and strength. We used four color models for skin color segmentation, these are RGB, $\mathrm{YC}_{\mathrm{b}} \mathrm{C}_{\mathrm{r}}, \mathrm{HSV}$, and LAB color models. After skin like pixels detection, we convert this segmented image into binary form. This binary image contains skin regions, but we don't know that, where is human face in segmented image. Next step of face detection after segmentation is based on knowledge of human face. Work of next step is to remove non human face skin area from segmented image, by using Knowledge-based methods or human face features [1]. In this paper, we are describing our algorithm in three section, section 1 presents 'Skin color segmentation', while section 2 presents 'Binary image formation' and section 3 presents 'Rejection of non face regions'. Fig. 1 is showing the flowchart of our proposed algorithm.
\end{abstract}
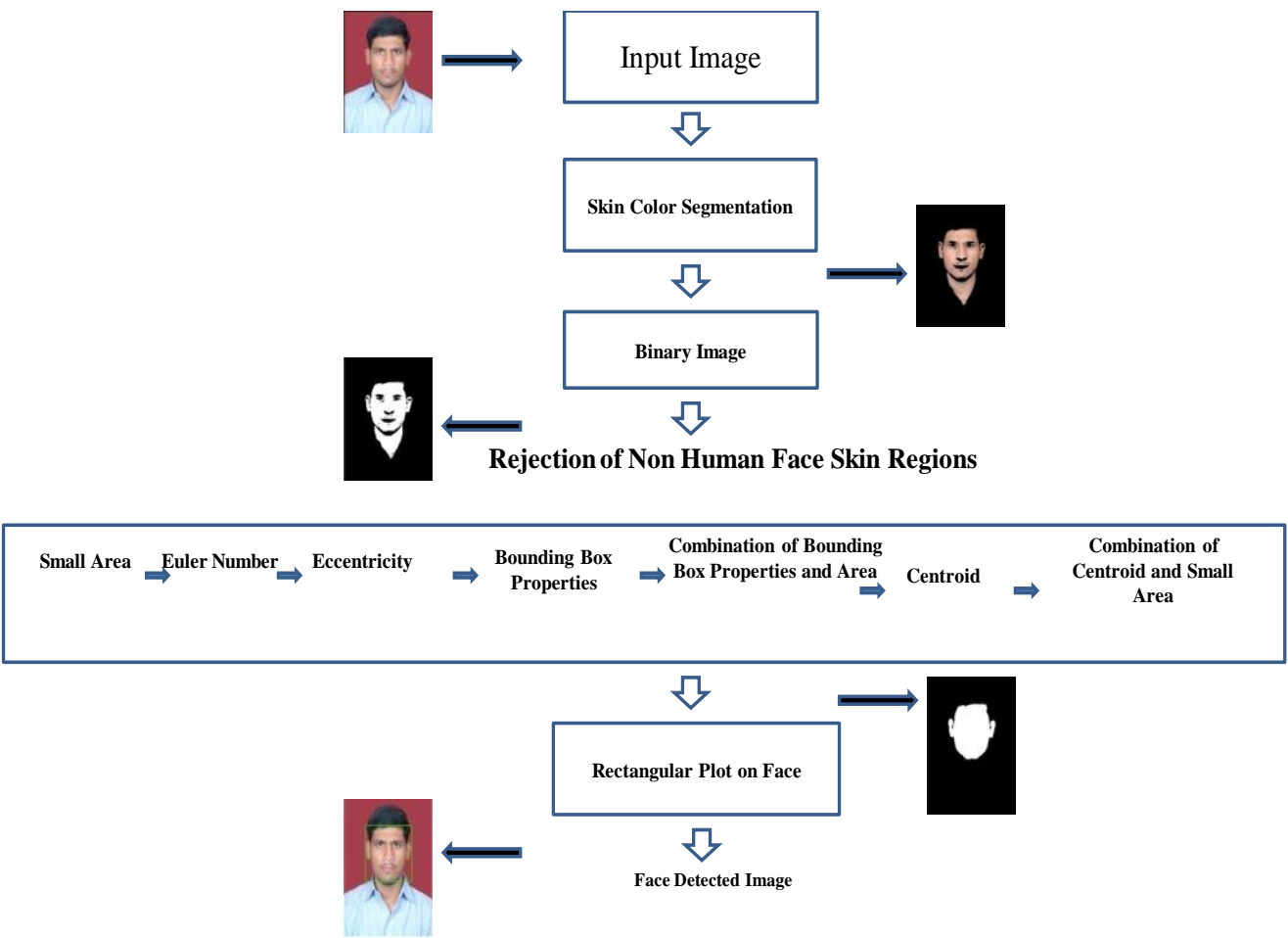

Fig 1: Proposed Face Detection Algorithm 


\section{SKIN COLOR SEGMENTATION}

Color is a useful piece of information for skin detection. The skin detection is the most common and first approach for detecting meaningful skin color [2], skin color detection may avoid exhaustive search for faces in an entire image. In this step, we describe that how non skin color is rejected from an Image so that the image may contains only skin like areas, which will be our skin color segmented image for further processing. From different type of color models, in HSV color model, Hue $(\mathrm{H})$ is not reliable for the discrimination task when the saturation is low, Also in $\mathrm{YC}_{\mathrm{b}} \mathrm{C}_{\mathrm{r}}$ color model, the distribution of skin areas is consistent across different races in the $\mathrm{C}_{\mathrm{b}}$ and $\mathrm{C}_{\mathrm{r}}$ color spaces, the RGB color model is lighting sensitive so Therefore, when we use different color models under uncontrolled conditions, and we get consequently result for skin color detection. The accuracy of skin detection depends on both the color model and the method of skin pixels classification or detection. Hence, the challenge problem is how to select color models that are suitable for skin pixel classifications under different varying conditions. In this thesis, there are four color models are used for skin color segmentation or detection of skin pixels. These are RGB, $Y_{\mathrm{b}} \mathrm{C}_{\mathrm{r}}$, and HSV and CIELAB color models. The combination of these color models overcomes all varying lighting conditions and changes in illumination, and it gives better result than individual color model result [3]. Figure 2 shows the combination of skin color segmentation.

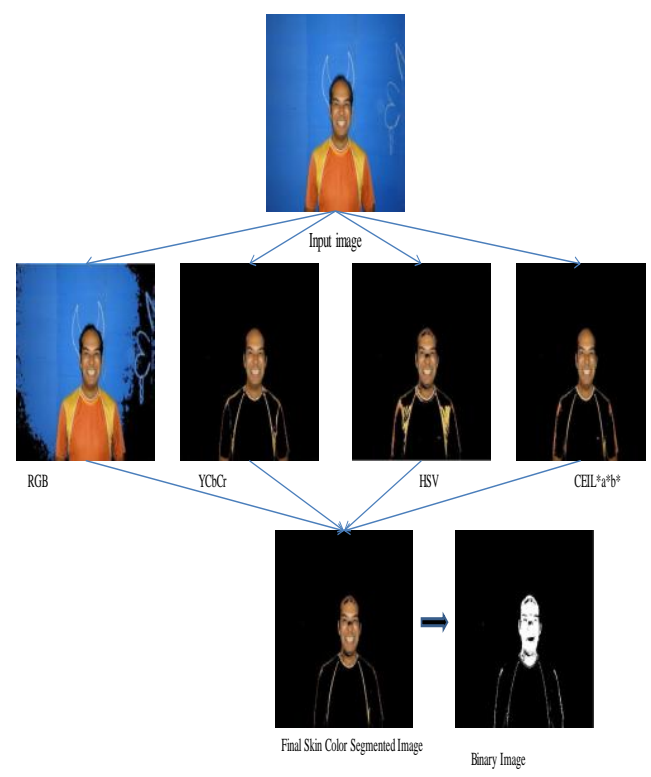

Fig 2: Skin color segmentation

\section{BINARY IMAGE FORMATION}

Skin color segmented image is contained only human skin like areas or regions, but we don't know that which region having exact human face, we need to send this image for further stage for rejection of non human face regions so that finally image contained only human face. If we take out this skin color segmented image for further processing, then our detection will be going to complex due to its $3 \mathrm{D}$ matrix version and different type of intensity values, so we need a binary image which is $2 \mathrm{D}$ matrix and having uniform intensity. A binary image is of class logical.
Binary images contain only 0's and 1's. Pixels with the value 0 are displayed as black, pixels with the value 1 are displayed as white. Skin regions are shown as white (logical ' 1 ') in binary image. The skin color segmented image, which is RGB image, is converted into binary image by using MATLAB function. First, we converted this segmented image into CIELAB image, and, we determine the gray threshold value of its 'A'part (2D matrix). By using, IM2BW MATLAB function, we convert this ' $a$ ' part into binary image.

\section{REJECTION OF NON HUMAN FACE SKIN REGIONS}

This step is the backbone of this algorithm. A binary image is contained skin regions, this step decides that, which region is most probable human face region by using human face features methods and region properties of human face. These are area of human face, Euler number, bounding box properties, centroid, eccentricity like oval estimation and combination features of these methods. A binary image of skin color segmented image with skin regions passes through this step one by one, then these stages of this step remove non human face like regions by using their properties which are fixed according to the human face. At finally, when binary image is totally passed from this step, image contains only highly probable human faces like regions. We retrieve the locations of these human faces from a filtered or passed binary image from this step. We plot rectangular around these highly probable human faces in original image. Here, we describing following non human face rejections methods:

\subsection{Small Area}

We calculate average area of human skin like regions of binary image, And compare this average area with each skin regions of binary image, if any skin regions is less than average value, then that skin region will be rejected $[4,5]$. Area of any skin region is calculated by counting no of skin region pixels. This method is helpful for removing very small skin regions from a binary image and, that binary image (after filtering from this method) proceed to next method or stage for removing non human face skin like regions.

\subsection{Euler Number}

Human faces contain some holes like eyes, eyebrows, a mouth, mustaches etc. the two eyes of human face are major holes for human faces. If any skin region doesn't contain any holes then, we can safely discard these type all skin region. The rejection is done by Euler number method $[5,6]$, the number of holes in a region is computed using the Euler number of region, the formula for Euler number is:

$$
\mathrm{E}=\mathrm{C}-\mathrm{H}
$$

$\mathrm{E}$ is Euler no. of skin region is the total number of object in the image minus total number of holes in the object.

$\mathrm{C}$ is the no. of connected component, and, $\mathrm{H}$ is no. of holes in a region. We can reject these type of skin regions which having Euler value greater than zero

\subsection{Eccentricity}

After rejection of non human face region from above method, now the image comes in this stage. Generally, shape of human face is likely to oval shape, so whose region which have shape probable like to oval shape these 
are not rejected by this method, and those regions whose shape likely to line are rejected [7]. For finding shape of skin regions, we used region properties based eccentricity MATLAB function, for each region, the function gives its eccentricity value. An ellipse (skin region) whose eccentricity is 0 is actually a circle, while an ellipse whose eccentricity is 1 is a line segment. The oval shape of a face can be approximated by an ellipse so we calculate Eccentricity of all skin connected regions and to discard all skin regions whose eccentricity greater than by 0.89905 .

\subsection{Bounding Box Properties of Human Face}

After passing from above methods, the binary image now passed from this stage, this stage reject non human face skin region based on height to width ratio [4, 6, and 8]. Generally, height to width ratio of skin regions is measurable factor because it is also big factor for rejecting non human face like regions. If height to width ratio of skin region is less than by threshold value, then this skin region will be discarding from class of probable human faces. Here we decided 1.902 threshold values for height to width ratio. For determining height to width ratio of each skin region, we used region properties based Bounding Box MATLAB function.

\subsection{Combination of Bounding Box Properties and Area}

Above said method 'Bounding Box Properties' work to maximum for rejecting non human face skin regions for those regions whom are not satisfy threshold value. In some typical cases, some region that don't belong probable human face class, but these regions satisfy height to width ration, and so these type of regions can't be rejected .for rejecting these type of regions, we introduced a method which is combination of Bounding Box method and area method [4]. We calculate skin region area bounded by bounding box and also calculate this bounding box area. Bounding box area is the multiplication of height and width of bounding box. Skin area (skin region pixels bounded by bounding box) is less than rectangular or bounding box area by two times, then this skin region will be rejected.

\subsection{Centroid}

Generally, the human faces are evenly distributed in the centre, means, that the human faces are not present in the side of images. Therefore, the centroid of a face region should be found in a small window centered in the middle of the bounding box.

We calculate y axis average centroid of a getting image after above methods. If centroids ( $Y$ coordinate) of skin regions are below and above by thresholds values, then these type of skin region will be rejected [4].

\subsection{Combination of Centroid and Small Area}

This method is the final stage of rejection of non human face like regions. This method is helpful to remove non human face like regions which are remain after above said methods. We again apply centroid method and area method separately but together.

\section{EXPERIMENTAL RESULTS}

Our face detection method was implemented with MATLAB 8.0. We tested our programming on more than 30 images which have single and multiple faces. We collected these images from our friends and internet. Following tables are showing the strength of our proposed algorithm

Table 5.1 Skin color classification results for RGB color space.

\begin{tabular}{|c|c|c|}
\hline $\begin{array}{c}\text { No. of } \\
\text { images }\end{array}$ & $\begin{array}{c}\text { False face detection } \\
(\boldsymbol{\%})\end{array}$ & $\begin{array}{c}\text { False dismissal } \\
(\boldsymbol{\%})\end{array}$ \\
\hline 41 & 45.93 & 32.43 \\
\hline
\end{tabular}

Table 5.2 Skin color classification results for $\mathrm{YC}_{\mathrm{b}} \mathrm{C}_{\mathrm{r}}$ color space.

\begin{tabular}{|c|c|c|}
\hline $\begin{array}{c}\text { No. of } \\
\text { images }\end{array}$ & $\begin{array}{c}\text { False face detection } \\
(\boldsymbol{\%})\end{array}$ & $\begin{array}{c}\text { False dismissal } \\
(\boldsymbol{\%})\end{array}$ \\
\hline 41 & 10.256 & 5.1282 \\
\hline
\end{tabular}

Table 5.3 Skin color classification results for HSV color space.

\begin{tabular}{|c|c|c|}
\hline $\begin{array}{c}\text { No. of } \\
\text { images }\end{array}$ & $\begin{array}{c}\text { False face detection } \\
(\boldsymbol{\%})\end{array}$ & $\begin{array}{c}\text { False dismissal } \\
(\mathbf{\%})\end{array}$ \\
\hline 41 & 15.3841 & 10.81081 \\
\hline
\end{tabular}

Table 5.4 Skin color classification results for CEILAB color space.

\begin{tabular}{|c|c|c|}
\hline $\begin{array}{c}\text { No. of } \\
\text { images }\end{array}$ & $\begin{array}{c}\text { False face detection } \\
(\boldsymbol{\%})\end{array}$ & $\begin{array}{c}\text { False dismissal } \\
(\boldsymbol{\%})\end{array}$ \\
\hline 41 & 10.132 & 5.1282 \\
\hline
\end{tabular}

Table 5.5 Skin color classification results for combination algorithm.

\begin{tabular}{|c|c|c|}
\hline $\begin{array}{c}\text { No. of } \\
\text { images }\end{array}$ & $\begin{array}{c}\text { False face detection } \\
(\boldsymbol{\%})\end{array}$ & $\begin{array}{c}\text { False dismissal } \\
(\boldsymbol{\%})\end{array}$ \\
\hline 41 & 4.44 & 2.3456 \\
\hline
\end{tabular}

Table 5.6 Detection rate of proposed face detection algorithm.

\begin{tabular}{|c|c|c|}
\hline $\begin{array}{c}\text { Method for face } \\
\text { detection }\end{array}$ & $\begin{array}{c}\text { No. of } \\
\text { images }\end{array}$ & $\begin{array}{c}\text { Detection result } \\
(\%)\end{array}$ \\
\hline $\begin{array}{c}\text { Skin color } \\
\text { segmentation }\end{array}$ & 41 & 20.0207 \\
\hline Face features & 41 & 38.46 \\
\hline Region properties & 41 & 46.158 \\
\hline Proposed algorithm & 41 & 92.30723 \\
\hline
\end{tabular}




\section{CONCLUSIONS}

The object of our paper is to develop an acceptable and efficient face detection algorithm to solve the challenge in face detection technology under varying conditions. In this paper, we applied a strong approach for face detection, the human skin areas are estimated by using skin color segmentation method. The skin color distribution in histogram map gives an idea to chose appropriate thresholds value for detecting skin pixels. Four color models are used for proposed skin color segmentation algorithm. This segmented image is converted into binary format image, this binary image has human face regions and non human face regions. Rejection of non human face regions methods decide which region is human face and which is not human face. The experimental results shows that, this algorithm justice the robustness of this method.

We want to increase its speed and to overcome on false face detection rate for increasing accuracy of the system in future. There are some recommendations for further improvements can be made in term of training examples for getting good results. The proposed Algorithm for this thesis detects only human faces in an image, After detecting human faces in an images there should be some extra features detection in future work, such as eyes, a nose and mouth detections in faces.

\section{ACKNOWLEDGMENTS}

We would like to thanks to all MANIT Electronics and communication engineering department staff and our friends.
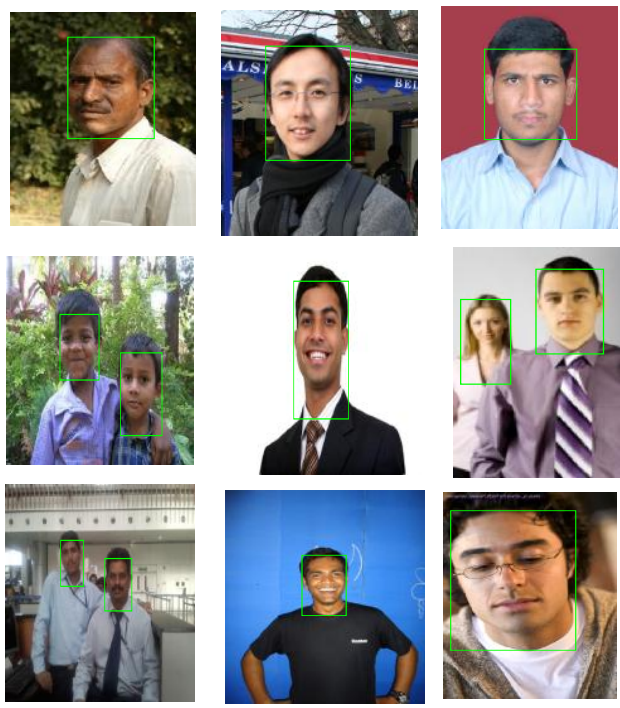

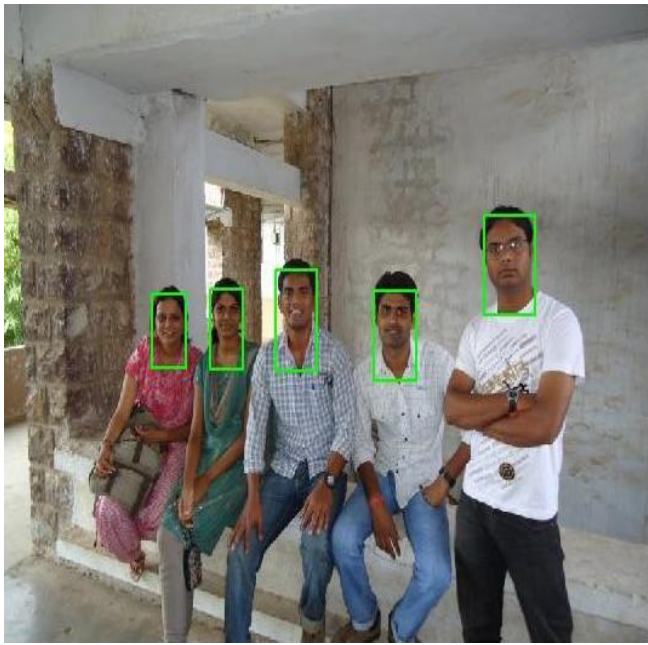

\section{REFRENCES}

[1] Ming-Hsuan Yang, member, IEEE, David J. Kriegman, Senior Member, IEEE, and Narendra Ahuja, Fellow, IEEE. "Detecting Faces in Images: A Survey", IEEE Transactions on Pattern Analysis and Machine Intelligence, vol. no. 1, January 2002.

[2] J. Liu and Y. H. Yang, "Multiresolution Color Image Segmentation", IEEE Transactions on Pattern Analysis and Machine Intelligence, Vol. 16, No. 7, pp. 689-700, 1994.

[3]. Sanjay Kr. Singh1, D. S. Chauhan2, Aayank Vatsa, Richa Singh "A Robust Skin Color Based Face Detection Algorithm", Tamkang Journal of Science and Engineering, Vol. 6, No. 4, pp. 227-234, 2003.

[4] R. Vijayanandh, Dr. G. Balakrishnan, "Human Face Detection Using Color Spaces and Region Property Measures", 2010 11th Int. Conf. Control, Automation, Robotics and Vision Singapore, December 2010.

[5] Murad Al Haj, Ariel Amato, Xavier Roca, and Jordi Gonzalez, "Finding Faces in Color Images through Primitive Shape Features", Computer Vision Center, Department d'Inform atica, Universitat Autonoma de Barcelona, 08193 Bellaterra, Barcelona, Spain, Institut de Robotica i Informatica Industrial (UPCCSIC), Barcelona, Spain.

[6] Waqar Mohsin Noman Ahmed khattak, "Face Detection Project", EE368 Digital Image Processing, Spring 02, 03, Department of Electrical Engineering Stanford University, 2002-2003.

[7] C . N. Ravi Kumar, Bindhu A, “ An Efficient Skin Illumination Compensation Model for Efficient Face Detection", 2010

[8] Yihu Yi, Daokui Qu, Fang Xu, "Face Detection Method Based on Skin Color Segmentation and Facial Component Localization", 2nd International Asia Conference on Informatics in Control, Automation and Robotics, 20 I0. 